\title{
The Prevalence and Risk Factors of Wrist Pain among Young Adults in UTAR during Movement Control Order: A Cross-Sectional Study
}

\author{
Kiruthika Selvakumar ${ }^{1}$, Liow Yi Sheng ${ }^{2}$ \\ ${ }^{1}$ Lecturer, Faculty of Medicine and Health Sciences, Department of Physiotherapy, \\ Universiti Tunku Abdul Rahman, Malaysia. \\ ${ }^{2}$ Year III Bachelor of Physiotherapy (Honours) Student, Faculty of Medicine and Health Sciences, \\ Department of Physiotherapy, Universiti Tunku Abdul Rahman, Malaysia. \\ Corresponding Author: Kiruthika Selvakumar
}

\begin{abstract}
Introduction: For the past few years wrist pain has been related to repetitive movement of the hand, and the cases has been increasing dramatically. There are numerous factors that causes wrist pain especially occupational factors. Different population has been investigated regarding work-related wrist pain such as, factory workers, programmers, and even gamers. Up to now, there is no data baseline about the prevalence and risk factors of wrist pain among young adults.

Objectives: To investigate the prevalence and risk factors of risk pain among young adults in University Tunku Abdul Rahman (UTAR) during Movement Control Disorder.

Methods: Questionnaire was adapted from Patient Rated Wrist Evaluation Score (PRWE), SelfDeveloped Demographic Data was used to determine prevalence and potential risk factors for wrist pain on young adults in UTAR during Movement Control Disorder, by using convenient sampling method.

Results: A total number of 144 participants had completed the questionnaire. Most of the participants were 20-25 years old. There are 100 female participants (69.4\%) and 44 male participants (29.9\%) who enrolled in this research. The prevalence of wrist pain among young adults in UTAR during Movement Control Disorder is relatively high according to PRWE score (71.53\%) while according to self-modified questionnaire there are 33 participants who felt the pain at the wrist (22.92\%).

Conclusion: Repetitive movement of the wrist and poor ergonomics of the hand are the factors that cause of wrist pain among young adults in UTAR.
\end{abstract}

Key Words: Prevalence, risk factors, wrist pain, young adults, Movement Control Disorder.

\section{INTRODUCTION}

Wrist pain is one of the musculoskeletal disorders which is affected by lengthy exposure to physical factors and an unexpected exertion like long term use of low amplitude, forceful, repetitive use of device, and even arthritis (1) (2). It can be due to sports participation, repetitive work, certain disease and condition, discord rand, fall on outstretched hand etc. In the nutshell, wrist pain can be due to many reasons and the most common causes include carpel tunnel syndrome (CTS), osteoarthritis, rheumatoid arthritis, De Quervain's disease, Kienböck's disease, repetitive motion syndrome, triangular fibrocartilage complex injury, wrist bursitis/tendonitis, ganglion cyst and wrist sprain. Some of the signs and symptoms that may cause wrist pain are, stiffness, swollen fingers, having difficulty to make a fistful or riveting objects, tingling sensation or numbness in the hands, pain numbness or tingling that worsen after evening, a soft clicking sound when moving 
the wrist, unexpected piercing pain at the hand, inflammation or redness at the wrist, warmness in a joint near the wrist and etc. For the past few years, repetitive movements of the wrist have been related with wrist pain, like the use of mouse and computer keyboards (3) (4). According to David et. al, their analysis confirms that within the cultural and psychosocial influences on disability research, disabling wrist hand pain was related with overall tendency to pain, comparative threats and population attributable fractions being complex which is known and so-called risk factors.

According to Melbourne Hand Rehab's, many of the citizens work from home during the Covid-19 pandemic and many of them enjoy the novelty initially. However, after 6 months, the novelty has lost its power and the number of patients who suffered due to the negative effects of a poorly setup home office has been increasing since then. The most common symptom encountered were sore wrist. When intend to extend wrist backwards or side to side an immense pain was felt. Instead of using computer which is place on the desk many people choose to use laptops, which is portable and light. This contributes to wrist pain, as they had been sitting on the couch or anywhere rather than using the desk with appropriate ergonomics. All of the awkward positions that we had leads to overloading of the specific tendons in our hand which leads to wrist pain eventually if the proper ergonomics were not practiced.

According to Fatima et. al (2020), mobile phones are all-pervading in this year and time. Those who spend most of their precious time on their smartphones may be causes a mild painful wrist and hand disorder. According to their research, the outcomes indicated that pain and disability at wrist was related to a huge number of students using cell phones. They find out that there is an important connection among spending lengthy hours on cell phones and an upsurge in disability and wrist pain. It seems like the extensive period of cell phones practice weakens the wrist joint. The monotonous actions and various locations of the wrist joint while using cell phone will affect the organizations of the wrist joint. In their recent research, there were $42 \%$ of the students spend not less than 5 hours on their cell phones which is stable with the job done previously. There was a nonsignificant relationship detected among screen dimensions of cell phone, pain and frailty of wrist joint. This was reinforced by the prior job about the relationship of screen dimensions and influence on wrist pain. In conclusion, those who spend more time on mobile devices tend to have a higher risk of having risk pain.

In Malaysia those who categorized as young adults are those who fall under the category of 20 to 44 years old. Those who are below 20 years old do have the risk of having risk pain as well, but it is comparatively low. On 18th March 2020, Movement Control Order, also known as (MCO) was documented by Malaysian Government due to the outburst of COVID19. According to Majlis Keselamatan Negara (2020), MCO consisted of few instructions to be followed by public. However due to the severity of the case, the Government decided to extend the Movement Control Order and name it as Enhanced Movement Control Order (EMCO) and Recovery Movement Control Order (RMCO). The prevalence of musculoskeletal disorder has been widely conducted nowadays. The musculoskeletal disorder usually covers the neck, shoulder, lower back etc. There are numerous studies with facts, numbers and data. However, when it comes to wrist pain among young adults specifically there is not much research about it especially in Malaysia. The objectives of this study were to investigate the prevalence and risk factor of wrist pain among young adults in Universiti Tunku Abdul Rahman (UTAR) during Movement Control Order (MCO). 


\section{MATERIALS AND METHODS}

A cross-sectional study is used to conduct this research to determine the prevalence and risk factors of risk pain among young adults in UTAR during movement control disorder. The research was conducted in UTAR Sungai Long Campus. The duration of study is 4 weeks starting from $14 / 11 / 2020$ to $11 / 12 / 2020$. During this study, convenient sampling method is used. The following is the formula used to estimate the sample size for cross sectional study suggested by the researchers by (Charan \& Biswas, 2013): $\mathrm{Z} 1-/ 2=1.96$ as in most of the studies, $\mathrm{P}$ values are considered significant less than $0.05, \mathrm{p}=$ estimated proportion of characteristics based on previous studies (0.50), $d=$ tolerated margin of error $(5 \%$ is chosen), $\mathrm{N}=$ population size. The participants in this research must be young adults currently in Universiti Tunku Abdul Rahman, Sungai Long campus. Furthermore, the participants must be at the age of 20 to 44 years old. The total participants estimated was 3123 participants. The exclusion criteria were young adults who cannot understand English and who don't have any devices which let them surf the net.

The study was approved by Scientific and Ethical Review Committee (SERC) of Universiti Tunku Abdul Rahman (UTAR) Sungai Long. Informed consent is obtained from all the participants before completing the questionnaire. Besides that, all participants are notified of the confidentiality of the information provided by them through Personal Data Information Statement. Participants were informed that they have the right to withdraw from the research at any moment. The researcher circulated the questionnaire through Google form. A brief explanation has been stated in the post about the purpose of the study and the procedure before they answered the questionnaire. After obtaining the agreement of participants for participating in the study, the participants are required to sign a consent form with a digital signature on it. Then, participants are prompted to answer all the questions step by step.

The questionnaire consists of 5 sections. First section represents the information of the study and also consent form for the participants. For the second section, it consists of the personal data protection statement to protect participants' information to be revealed. The third section is about the socio-demographic information and risk factors of wrist pain. The fourth and fifth section is about patient rated wrist evaluation score (PRWE), pain and function. This combined questionnaire which includes consent form, personal data protection statement, Self-Developed Demographic Data, and patient rated wrist evaluation score (PRWE) take approximately only 8 to 10 minutes to complete. As mentioned, the third section is about Socio-demographic information and risk factors of wrist pain. The question such as age, is used to spell out all participants who do not fit the inclusion criteria of this study. Question 4 to question 20 is to determine the risk factors wrist pain. Such as, gender, ethnicity, dominant hand, how frequent the participants smoke, drink, spend their time on their devices and even what kind of devices they are using. Furthermore, questions like past history and medical history have also been included in this questionnaire. Section 3 contains both open ended and close ended questions.

Section 4 is related to patient rated wrist evaluation score (PRWE) and consist of 5 questions. The participants were requested to rate the average of pain in their wrist over the past week by choosing the number that best describes their pain on a scale of 0 to 10 . A zero (0) means that they did not have any pain and a ten (10) means that they had the worst pain they have ever experienced or that they could not do the activity because of pain. Section 5 , the last section of the questionnaire consists of 10 questions. Similar to section 4 , participants were asked to rate the amount of difficulty when performing each of the item listed in section 5 on a scale of zero to ten. Example, 
turn a doorknob using their affected hand, cut a meat, push up from a chair, carry a 10 lb. and object and household works.

\section{Statistical analysis}

Data analysis was performed with IBM SPSS Statistics 23. The alpha level set to $\mathrm{p}<0.05$ to statistically significant. Demographic data, the risk factors and the occurrence of wrist pain among young adults were analyzed using descriptive statistics method. The Cronbach's Alpha of the reliability statistics for this study is set at 0.74 .

\section{RESULTS}

\section{Social Demographic}

A total number of 144 participants had completed the questionnaire. 4 participants were not qualified to be included in the study as they do not meet the inclusion criteria. All participants originated from UTAR Sungai Long Campus. The response obtained are $100 \%$ through online survey administration. Of 132 participants 91.7\% were UTAR student $3.5 \%$ UTAR Lecturer, 2.8\% were UTAR staff and 2.1\%
UTAR Alumni. According to the survey, most of the participants are in the range of 20-25 years old, with total of 128 participants (88.9\%), followed by 26-30 years old with 6 participants (4.2\%). Next, there are 3 participants who fall in the category of $31-40$ years old (2.1\%). There are no participants who aged from 41-44 years old or more than 44 years old. Majority of the participants were Chinese $(n=127,88.2 \%)$. There was total 13 Indian participants (9.0\%) whereby Malay participants consist of 4 (2.8\%).

\section{Risk factors for wrist pain}

\subsection{Gender}

100 female participants (69.4\%) and 43 male participants (29.9\%) enrolled in this research. There is 1 participant whose gender fall under the category of 'other' $(0.7 \%)$.

\subsection{Dominate hand}

There were 132 number of participants who is left-handed (91.7\%) and 12 number of participants who is righthanded (8.3\%).

\subsection{Related risk factors}

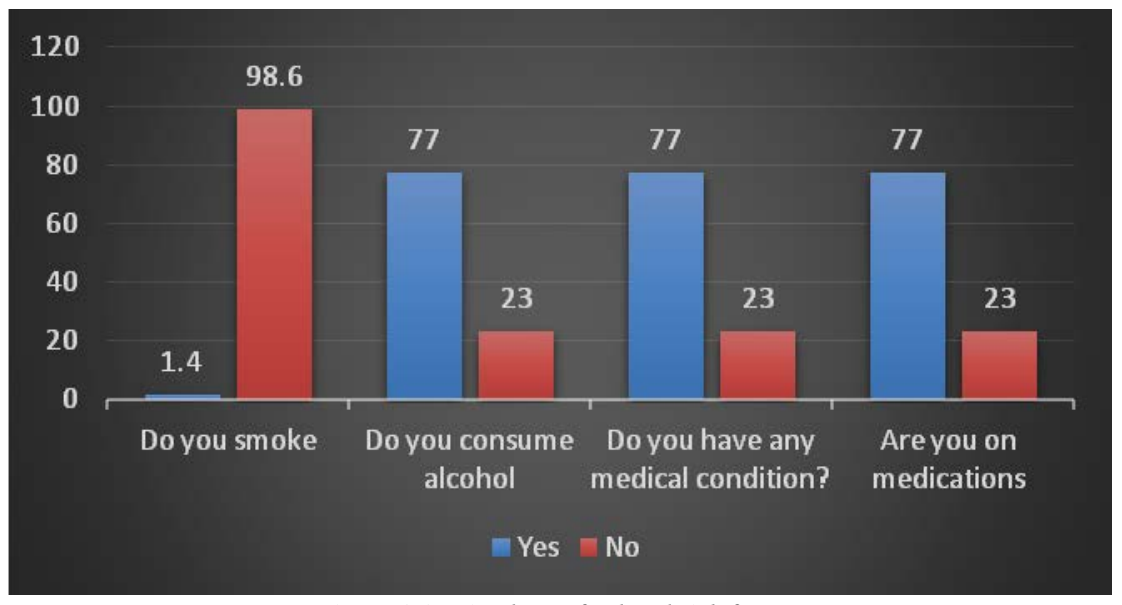

Figure 2.3: Pie chart of related risk factors

According to Figure 2.3, only 2 participants who actually have the habit of smoking (1.4\%) while the rest of the participants doesn't not have the habit of smoking ( $n=142,99 \%)$. There were 111 participants who does not consume alcohol (23\%), and there are 33 number of participants who consume alcohol (77\%). Most of the participants only consume alcohol once per month $(n=5,21 \%)$ and some participants mentioned they only drink occasionally $(n=5,21 \%)$. There are also participants who only drink twice per month $(n=4,13 \%)$ and once per week $(n=3,12 \%)$. 
The rest of the participants seldom consume alcohol $(\mathrm{n}=2,8 \%)$, only drink once per month $(\mathrm{n}=1,4 \%)$ and even twice a year $(\mathrm{n}=1,4 \%)$. According to the Figure 4.1.9 (b), there are only 4 person who have preexisting medical conditions (3\%), 3 of them have asthma while another participant has a scoliosis. There are 140 participants who doesn't have any pre-existing medical condition (97\%). According to the Figure 4.1.10 (b) only 3 people is on medication (2\%) and the rest is not on medication, $(n=141,98 \%)$.

\subsection{Devices}

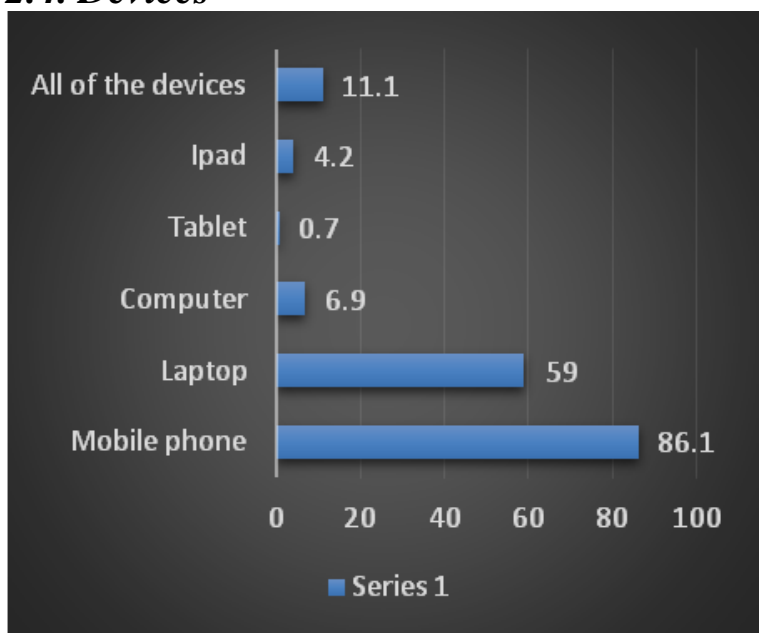

Figure 2.4: Bar chart on the devices the participants use the most

\subsection{Hours the participants spend with their devices}

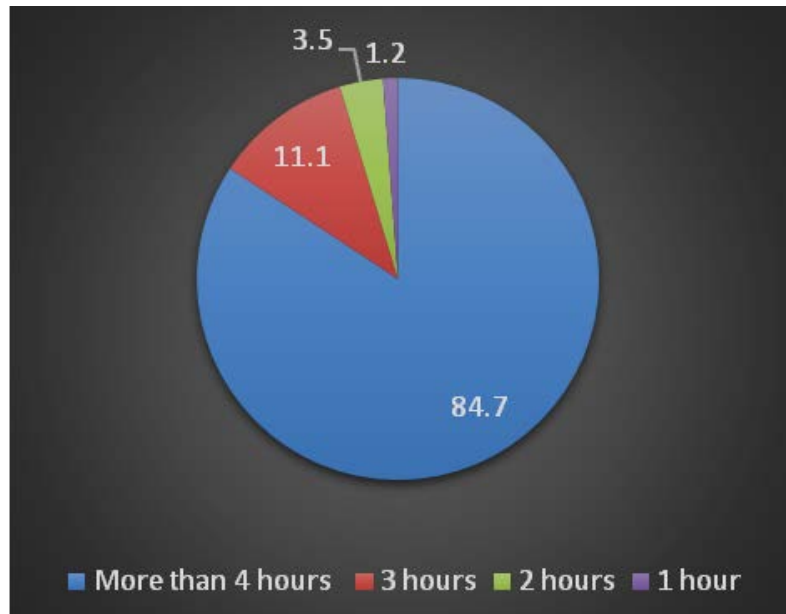

Figure 2.5: Pie chart how many hours the participants spend with their devices

According to Figure 2.5 there are 122 participants who spend more than 4 hours a day with their devices (84.7\%) and
16 participants who spend their time using their device for 3 hours (11.1\%). Next there are 5 participants who spend 2 hours with their device (3.5\%) and 1 person who actually spend only 1 hour on their device $(0.7 \%)$.

\subsection{Purpose of use the device}

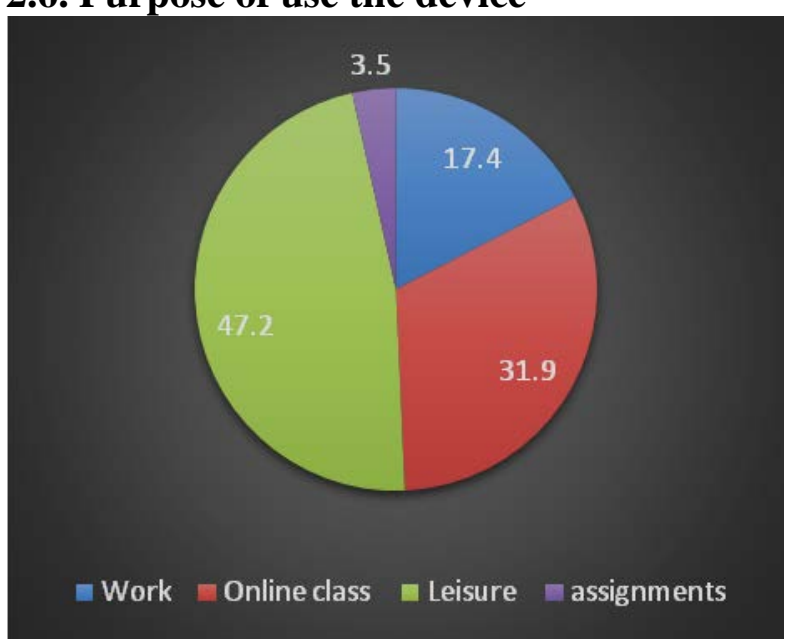

Figure 2.6: Pie chart on the purpose the participants use the device for

According to Figure 4.1.12 (b) above we can clearly see that majority of the participants use their device for leisure $(n=68,47.2 \%)$ and for online classes $(n=46$, $31.9 \%$ ). Next there are 25 number of participants who use their device for working purpose (17.4\%). Last and not least there are participants who used their devices for assignments as well $(n=5,3.5 \%)$.

\subsection{Tendency of breaks}

135 participants do take breaks in between when they are using their device (93.8\%). There are 9 people who doesn't take breaks in between when using their device $(6.3 \%)$.

\subsection{Duration of breaks the participant had}

There are $47 \mathrm{f}$ participants who take breaks more than 30 minutes (32.6\%) and 31 participants who take 10 minutes break in between (21.5\%). Next, there are 28 participants who take 5 minutes break (19.4\%) and 23 participants who take 15 minutes in between (16\%). Lastly there are 15 number of participants who take 20 minutes break in between (10.4\%). 


\subsection{Recent falls the participant had}

There are 129 participants who doesn't have any recent fall between $1 / 5 / 2020$ to $10 / 10 / 2020(89.6 \%)$ and 15 participants had falls between $1 / 5 / 2020$ to 10/10/2020 (10.4\%).

\subsection{Presence of lump at the wrist}

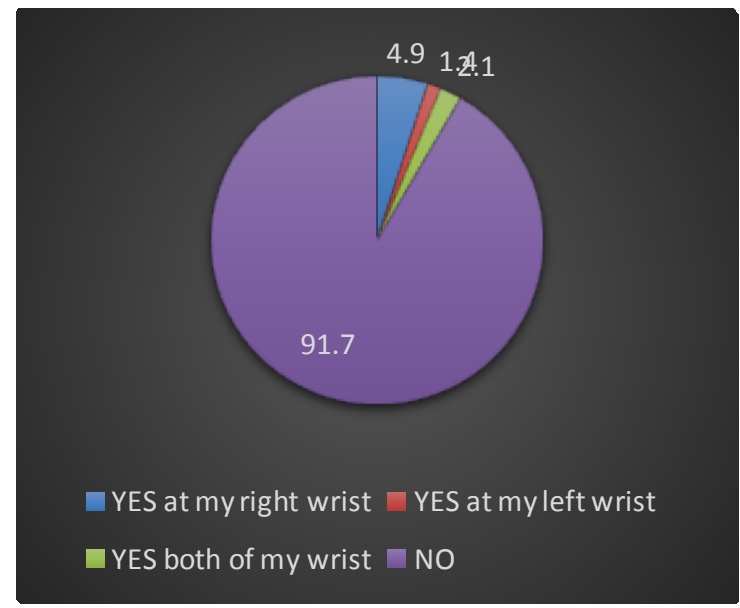

Figure 3.0: Pie chart on the presence of lump at the wrist among the participants

According to the Figure 3.0, there are 132 number of participants who does not have any visible/palpable lump at their wrist which causes pain (91.7\%). Next there are 7 participants who had a visible/palpable lump at their right wrist which causes pain (4.9\%) and 2 participants who had a visible/palpable lump at their left wrist which causes pain (1.4\%). Lastly there are 3 participants who had a visible/palpable lump at both of their wrist which causes pain (2.1\%).

\subsection{Presence of pain at the lunate region}

According to the Figure 4.1.18 (b) above we can clearly see that there are 128 number of participants who doesn't feel the pain when they press the lunate region gently (90.1\%). However, there are 14 number of participants who felt the pain when they press the lunate region gently $(9.9 \%)$.

\subsection{Presence of stabbing pain/tingling sensation/pricking sensation at the tip of the fingers or the thumb}

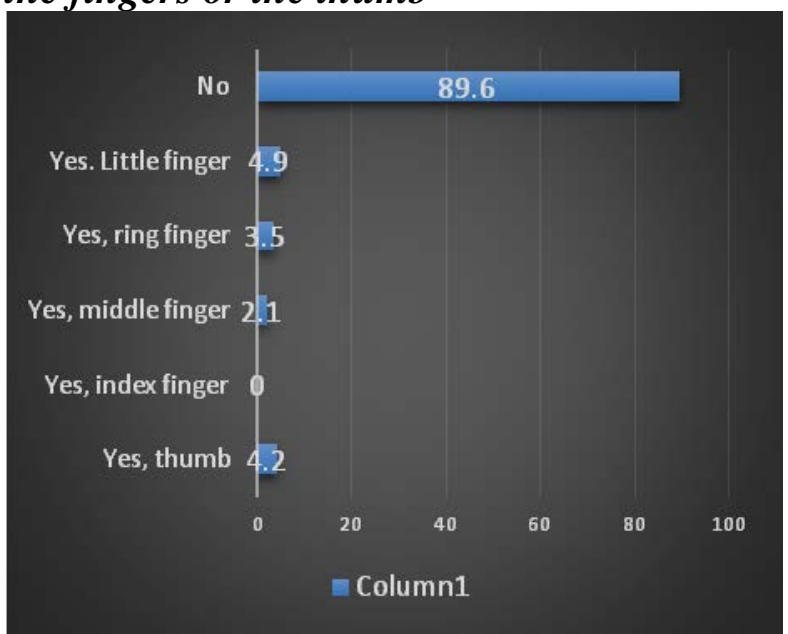

Figure 3.2: Bar chart on the presence of stabbing pain/tingling sensation/pricking sensation at the tip of the fingers or the thumb among the participant

\section{Patient Rated Wrist Evaluation Score (PRWE score)}

\subsection{Pain}

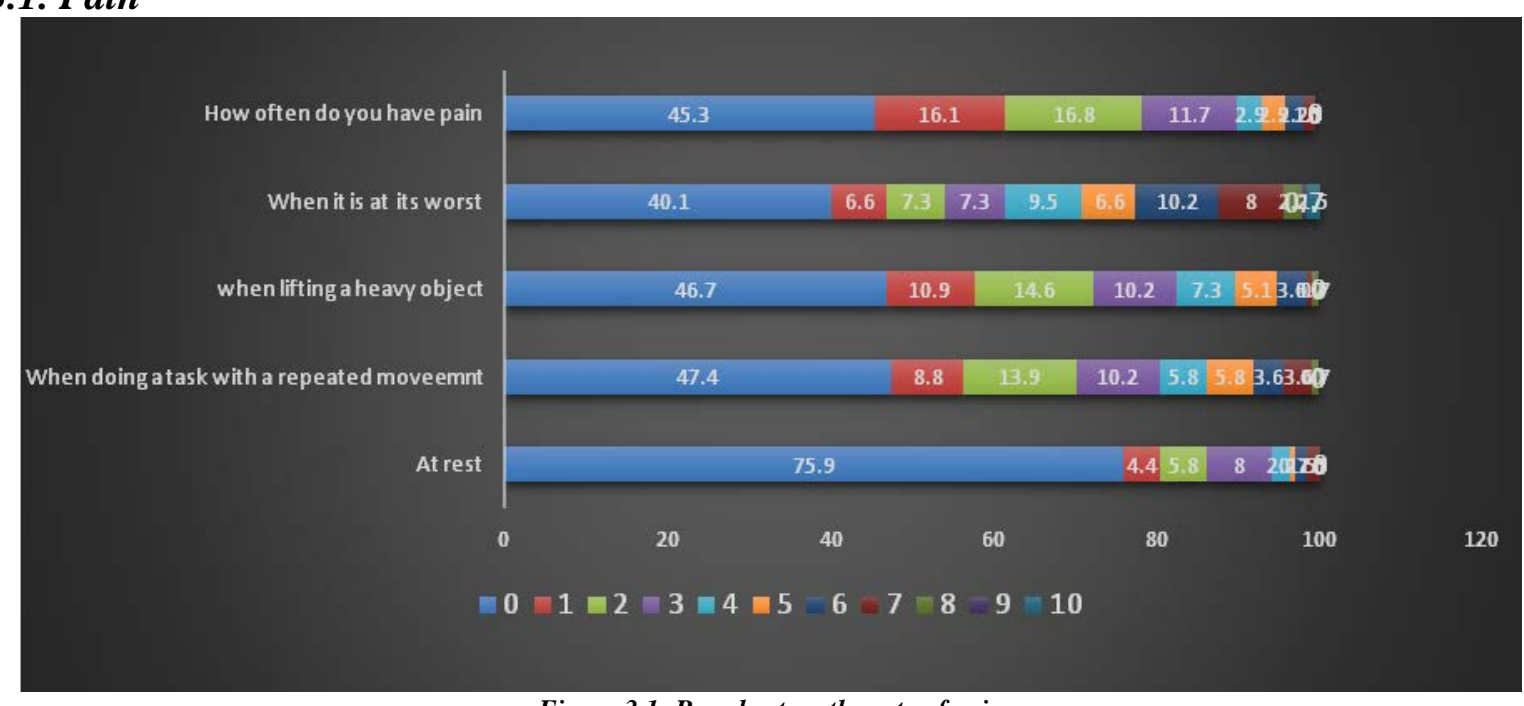

Figure 3.1: Bar chart on the rate of pain 
There are 104 participants who don't feel any pain at the wrist during resting $(75.9 \%)$. There are 6 participants rate the pain as $1(4.4 \%), 8$ participants rate the pain as $2(5.8 \%), 11$ participants rate the pain as $3(8 \%), 3$ participants rate the pain as $4(2.2 \%), 1$ participant rate the pain as $5(0.7 \%), 2$ participants rate the pain as $6(1.5 \%)$ and 2 participants who rate the pain as 7 (1.5\%). There was no participant who rate the pain as $8 / 9 / 10(n=0,0.0 \%)$.

According to Figure 3.1 there are 65 participants who don't feel the pain when doing a task with a repeated wrist movement (47.4\%). There are 12 participants rate the pain as 1 (8.8\%), 19 participants rate $2(13.9 \%), 14$ participants rate 3 (10.2\%), 8 participants rate 4 (5.8\%), 8 participants rate 5 (5.8\%), 5 person rate 6 (3.6\%), 5 participants who rate 7 (3.6\%) and 1 participant who rate $8(0.7 \%)$.

According to Figure 3.1 "when lifting a heavy objects”, 64 participants don't feel the pain (46.7\%). 15 participants rate the pain as 1 (10.9\%), 20 participants rate as $2(14.6 \%), 14$ participants rate 3 (10.2\%), 10 participants rate the pain as 4 (7.3\%), 7 participants rate the pain as 5 (3.6\%), 1 person rate the pain as $7(0.7 \%)$ and I participant who rate the pain as 8 $(0.7 \%)$.
According to the Figure 3.1 "when it is at worst" there are 55 participants who don't feel the pain (40.1\%). There are 9 participants rate the pain as 1 (6.6\%), 10 participant rate 2 (7.3\%), 10 participant rate $3(7.3 \%), \%), 13$ participant rate 4 (9.5\%), 9 participant rate 5 (6.6\%), 14 participant rate 6 (10.2\%), 11 participant rate $7(8.0 \%), 3$ participant rate $8(2.2 \%), 1$ participant rate $9(0.7 \%)$ and 2 participant rate $10(1.5 \%)$.

According to the Figure 3.1 "how often do you feel pain" there are 62 participants who don't feel the pain (45.3\%). There are 22 participant rate 1 (16.1\%), 23 participants rate 2 (16.8\%), 16 participants rate 3 (11.7\%),4 participant rate 4 (2.9\%), 4 participant rate 5 (2.9\%), 3 participant rate $6(2.2 \%), 2$ participant rate 7 (1.5\%) and 1 participant rate 10 (0.7\%).

\subsection{Specific activities}

According to Figure 3.2, there are 111 participants who don't feel the pain when turning a doorknob using their affected hand (81.0\%). 12 participants rate the pain as 1 (8.8\%), 4 participant rate it as 2 (2.9\%), 4 participant rate it as 3 (2.9\%), 1 participant rate it as $4(0.7 \%), 2$ participant rate it as 5 (1.5\%), 2 participant rate it as 7 $(1.5 \%)$ and 1 participant rate it as $8(0.7 \%)$.

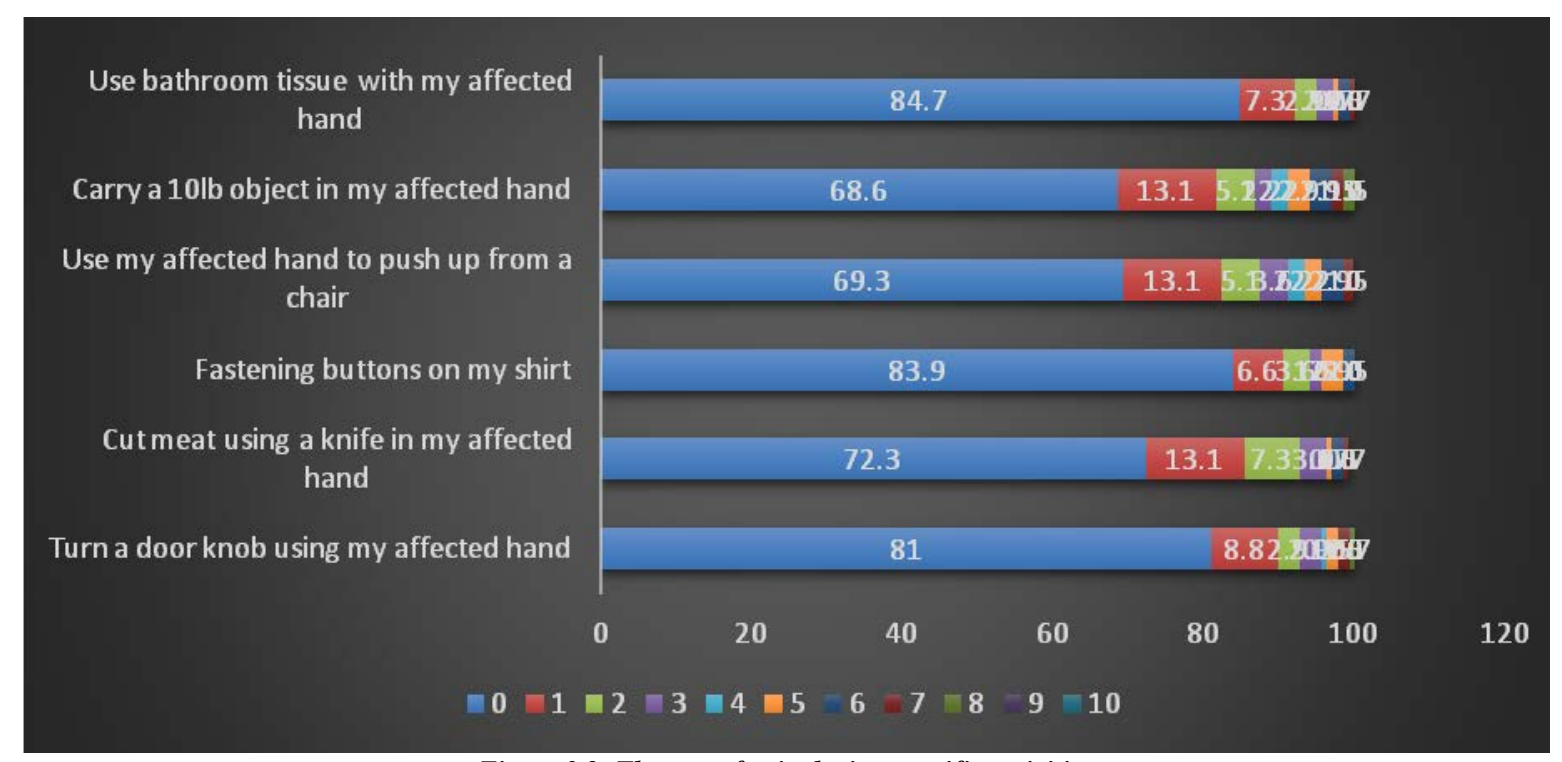

Figure 3.2: The rate of pain during specific activities

According to the Figure 3.2, 99 number of participants don't feel the pain
72.3\% when cutting the meat using a knife in their affected hand. There are 18 
participant who rate the pain as 1 when cutting the meat using a knife in their affected hand (13.1\%), 10 participant rate it as $2(7.3 \%), 5$ participant rate it as 3 (3.6\%), 1 participant rate it as5 (0.7\%), 2 participant rate it as $6(1.5 \%) 1$ participant rate it as $7(0.7 \%)$ and 1 participant rate it as $10(0.7 \%)$.

According to the Figure 3.2, there are 115 number of participants who don't feel the pain when fasten buttons on their shirt (83.9\%). There are 9 participants who rate the pain as 1 when fasten their shirt (6.6\%), 5 participants who rate the pain as 2 (3.6\%), 2 participants who rate the pain as $3(1.5 \%), 4$ participants who rate the pain as5 (2.9\%) and 2 participants who rate the pain as $6(1.5 \%)$ when fastening their shirt.

According to the Figure 3.2, there are 95 participants who don't feel the pain when the participants use their affected hand to push up from a chair (69.3\%). There are 18 number of participants rate the pain as $18(13.1 \%), 7$ participants rate the pain as 1 (13.1\%), 7 participants rate the pain as $2(5.1 \%), 5$ participants rate the pain as 3 (3.6\%), 3 participants rate the pain as $4(2.2 \%), 3$ participants rate the pain as $5(2.2 \%), 4$ participants rate the pain as6 $(2.9 \%)$, and 2 participants rate the pain as 7 (1.5\%).

According to the Figure 3.2, there are 94 number of participants who don't feel

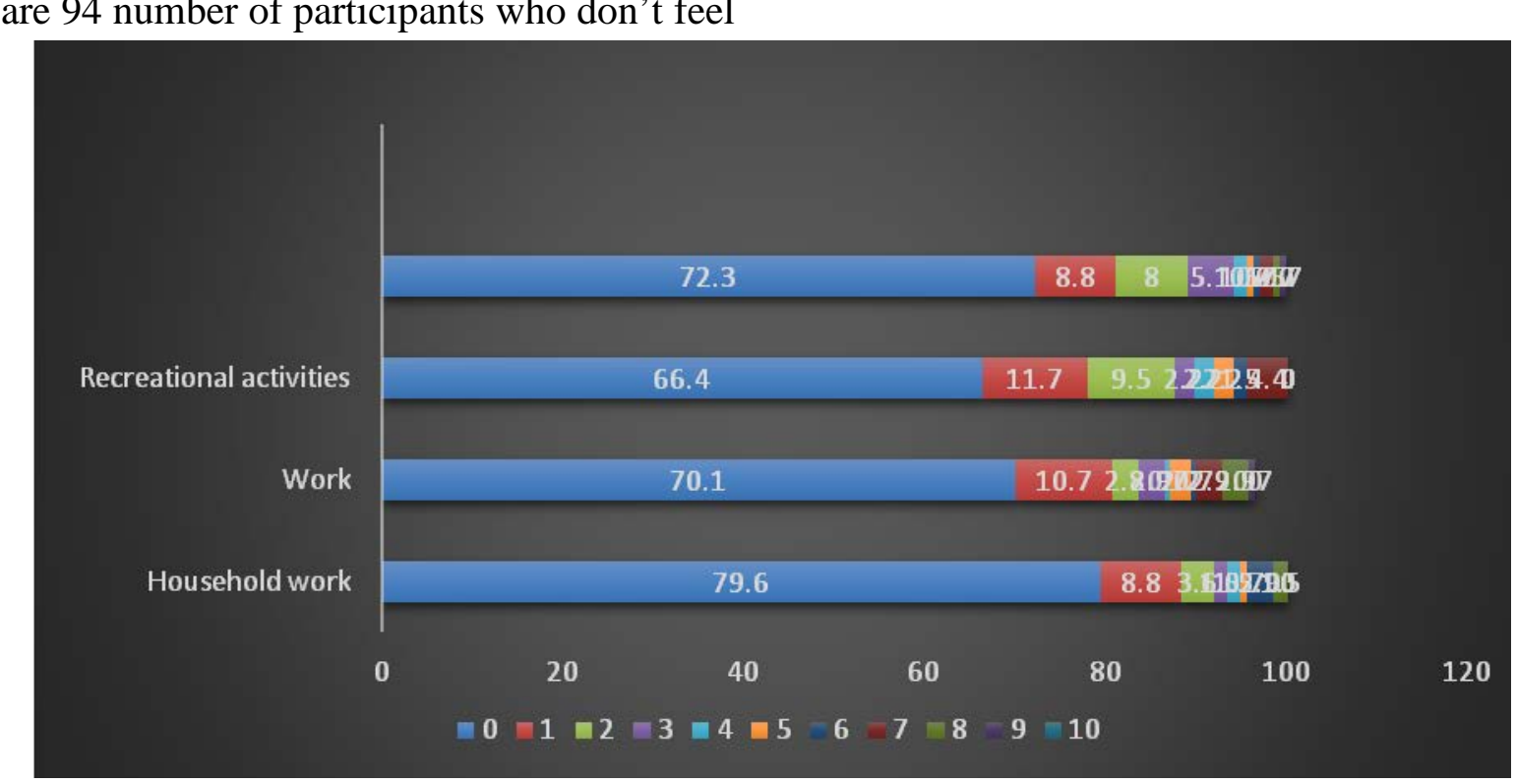

Figure 3.3. Bar graph on the rate of pain when the participants do usual activities the pain (68.6\%). There are 187 participants who rate the pain as 1 when carrying a 10 IB object in their affected hand (13.1\%), 7 participants rate their pain as $2(5.1 \%), 3$ participants rate their pain as $3(2.2 \%), 3$ participants rate their pain as $4(2.2 \%), 4$ participants rate their pain as $5(2.9 \%), 4$ participants rate their pain as $6(2.9 \%), 2$ participants rate their pain as $7(1.5 \%)$ and 2 participants rate their pain as $8(1.5 \%)$.

According to the Figure 3.2, there are 116 number of participants who don't feel the pain (84.7\%) when they use bathroom tissue with their affected hand. There are 10 participants rate the pain as $1(7.3 \%), 4$ participants rate the pain as $2(2.9 \%), 3$ participants rate the pain as $3(2.2 \%), 1$ participant rate the pain as $5(0.7 \%), 2$ participants rate the pain as $6(1.5 \%)$ and 1 participant rate the pain as $7(0.7 \%)$.

\subsection{Usual Activities}

According to Figure 3.3, there are 109 participants who don't feel the pain when they deal with their personal activities (79.6\%). There are 12 participants rate the pain as $1(8.8 \%), 5$ participants rate the pain as $2(3.6 \%), 2$ participants rate the pain as $3(1.5 \%), 2$ participants rate the pain as $4(1.5 \%), 1$ participant rate the pain as $5(0.7 \%), 4$ participants rate the pain as $6(2.9 \%)$, and 2 participants rate the pain as $8(1.5 \%)$. 
According to the Figure 3.3, there are 96 number of participants who don't feel the pain when they deal with their household work (70.1\%). However there are 15 participants rate the pain as 1 (10.9\%), 8 participants rate the pain as 2 (5.8\%), 4 participants rate the pain as 3 (2.9\%), 1 participant rate the pain as 4 $(0.7 \%), 3$ participants rate the pain as 5 (2.2\%), 1 participants rate the pain as 6 $(0.7 \%), 4$ participants rate the pain as 7 (2.9\%), 4 participants rate the pain as 8 (2.9\%) and 1 participant rate the pain as 9 (0.7\%).

According to the Figure 3.3, there are 91 number of participants who don't feel the pain when they deal with their work (66.4\%). However, there are 16 participants rate the pain as $1(11.7 \%), 13$ participants rate the pain as $2(9.5 \%), 3$ participants rate the pain as $3(2.2 \%), 3$ participant rate the pain as $4(2.2 \%), 3$ participant rate the pain as $5(2.2 \%), 2$ participant rate the pain as 6 (1.5\%), and 6 participant rate the pain as 7 (4.4\%) when they deal with their work.

According to Figure 3.3, there are 99 participants who don't feel the pain when they deal with their recreational activities (72.3\%). However, there are 12 number of participants rate the pain as 1 (8.8\%), 11 participants rate the pain as 2 (8.0\%), 7 participants rate the pain as 3 (5.1\%), 2 participants rate the pain as 4 (1.5\%), 1 participant rate the pain as 5 $(0.7 \%), 2$ participants rate the pain as 7 (1.5\%), 1 participant rate the pain as 8 $(0.7 \%)$ and 1 participant rate the pain as 9 (0.7\%).

\section{DISCUSSION}

This study determined and investigated the prevalence of wrist pain among young adults in University Tunku Abdul Rahman by using self-modified questionnaire and Patient Rated Wrist Evaluation Score (PRWE). With all the selfmodified questionnaire (22.92\%, $\mathrm{n}=33$ ) and Patient Rated Wrist Evaluation Score (PRWE) (71.53\%, n=98), in this study we can conclude that wrist pain is common among young adults. This study suggest that wrist pain is not only seen in those who partake in physically demanding activities like manual laborers and sportspeople as mentioned in other articles, it can also happen in general population as well, the results are in line with other studies. In 2018 a research has been done in Malaysia to determine the prevalence of musculoskeletal disorder among office workers in Lembaga Tabung Haji Kuala Lumpur. The prevalence of musculoskeletal disorders among office workers was $82.3 \%$ and the percentage of wrist pain is $30.6 \%$ in their study, thus we can see that wrist pain is affected by occupation and the ergonomics of it and in the matter of fact it is very common nowadays as I had mention earlier. According to Ferguson and his teammates (2019), 'wrist pain is highly prevalent in groups who devoted themselves in substantially challenging activities from each day such as manual laborers and sports related people (5)' As they discovered that the median prevalence of wrist pain in the general population and non-manual workers was 6 and 4.2\% within the short term and within the medium term which is more than one week and within a year.

Age is one of the factors studied in many research as a suggestion with musculoskeletal pain. 'Aging will cause degeneration of cartilage and tendon which cause a greater risk of developing workrelated musculoskeletal disorders' (6). An institute for Work \& Health (2013) have analyzed a total of 27,000 Canada workers on the relationship of age and musculoskeletal disorders who age between 35 to 44 years old have the highest risk of musculoskeletal injury. According to the study, almost $90 \%$ of the participants are from the range of 20 to 24 years old while other participants who's aged between 2544 is very less. Thus, a valid correlation could not be generated and validated. However, those who aged between 25-44 years old tend to have wrist pain in this study (95\%). According to Yvone and her teammates, (2020) women have a higher 
perception of pain and sensitivity towards pain. Women are more susceptible to pain compared to men. According to Gregori Surgery Center, women tend to have Carpel Tunnel Syndrome which will eventually leads to wrist pain. Besides according to American Thyroid Association, women are more likely to develop thyroid disorder, particularly five to eight times more likely compared to men. Thyroid disorder is one of the key factors that causes Carpel Tunnel Syndrome (CTS). In this study we can see that the percentage of females to have wrist pain is much higher than male in UTAR Sungai Long as in relation with previous studies.

Young adults in Universiti Tunku Abdul Rahman in Malaysia are affected by wrist pain during Movement Control Order. This is due to the repetitive use of wrist. During Movement Control Order, the citizens of Malaysia worked from home and even have online classes instead of physical classes except those providing essential services and items (Tang, 2020). Working from home and online classes require the citizens to surf the net, thus they have to spend more time with their devices such as laptops, mobile phones, computer, iPad, tablet and etc in. In this research there are 122 number of participants $(84.7 \%)$ who actually spend more than 4 hours on their devices, 16 number of participants spend 3 hours on their devices (11.1\%), 5 participants who spend 2 hours (3.5\%) and 1 participant who spend only 1 hour on his/her device $(0.7 \%)$. It means that, young adults in University Tunku Abdul Rahman are having a wrist pain mainly due to repetitive use of the wrist and of course with the wrong ergonomics. As known, surfing the net required us to type; prolong typing will strain the wrist; wrong ergonomics worsen the condition of the wrist. "Adoption of stressful postures for long duration might cause musculoskeletal disorders in different parts of the body" (Paj, et. al, 2017). "Typing using a keyboard tilted at a 20 degree will leads to a change in the median nerve (Carpel Tunnel Syndrome)"(7)

There are 135 number of participants claimed that they did they a break in between when they are using their device (93.8\%). However, there are only 47 number of participants who claimed that they had a break which is more than 30 minutes in between (32.6\%). 31 participants claimed that they had a 10-minute break (21.5\%), 5 minutes break ( $\mathrm{n}=28,19.4 \%), 15$ minutes break $(n=23,16 \%)$ and even 20 minutes break $(n=15,10.4 \%)$. Breaks in between do help in preventing the wrist from getting strain, but it is clearly not enough, as they are having wrist pain currently at the end of the day. Next, the condition gets even worst as they involved in sports and recreational activities, because they strain their wrist even more during this period of time. Some of the sport activities that had are, power lifting, badminton, swimming, boxing, basketball, futsal and even fishing. In normal circumstances, these activities do benefit us. However, during this pandemic COVID-19, we had already strained our wrist in different kind of forms, and ways and even with different kind activities. Thus, it doesn't benefit us anymore for the time being.

There are different sign and symptoms that shows that the condition has been worsen. Example, there are 8.3\% $(n=12)$ of participants claimed that there are a visible/palpable lumps at the wrist which causes pain, 9.9\% $(n=14)$ of participants claimed that they feel the pain when they press the lunate region, $10.4 \%(n=15)$ of participants feel a stabbing pain/tingling sensation/pricking sensation at the tip of their fingers or at their thumb and 33 number of participants (24.1\%) even feel the pain during rest. All these signs and symptoms suggest that they had carpel tunnel syndrome, kienbock's disease, tenosynovitis de quervain, tennis elbow etc. "Tenosynovitis de quervain is due to repetitive, forceful and ergonomically stressful labor, hormonal influence, trauma and from anatomical variation” (8). 
"Kienböck disease is believed as a result of repetitive trauma to the lunate due to occupational activity" (9). "Carpel Tunnel Syndrome is a condition due to compression of the median nerve which causes, numbness, tingling sensation in the patient's hand" (10). This statement supports our studies, as the participants in our studies do feel pain at the wrist when buttoning their shirt and push up from a chair. According to Natalie Philips, Gout is cause by an accumulation of uric acid. Uric acid usually will dissolve in blood and removed from the body through urination (11). This excess uric acid will deposit in the joints which causes pain and swelling. This will commonly occur at the wrist, ankle, knee and feet. One of the factors which causes gout is by consuming too much alcohol. Burger's disease also e called thromboangiitis obliterans can caused by smoking. Buerger's disease affects blood vessels in the body.

According to the data collected, wrist pain is not associated with smoking and drinking, as the data that have collected has no significant difference. There are $98.6 \%$ of the participants who is not smoking $(n=142)$ and 111 number of participants who are not drinking (23.0\%) according to the study and yet majority of the participants are having pain at the wrist. This may be due to the frequency of the participants in consuming alcohol. Most of the participants only consume alcohol once per month $(n=5,21 \%)$ and some participants mentioned they only drink occasionally $(n=5,21 \%)$. There are also participants who only drink twice per month $(n=4,13 \%)$ and once per week $(n=3,12 \%)$. The rest of the participants seldom consume alcohol $(n=2$, $8 \%)$, only drink once per month $(n=1,4 \%)$ and even twice a year $(n=1,4 \%)$.

\section{Limitations}

There are few limitations being found in this cross-sectional study. Due to the pandemic covid-19 and the Movement Control Order, citizens of Malaysia have been very careful with each other. Physical forms were distributed; however, it has been rejected by all parties. Thus, a convenient sampling method has been used to run throughout the study. Therefore, sample bias was present. Next, due to the pandemic covid-19 also, the respondent is relatively small in size which affects the results. Besides the sample size in this study is undetermined as some of the faculty in University Tunku Abdul Rahman could not provide the data who is categorized as young adults due to privacy issues. Besides, this study only recruited young adults in in Universiti Tunku Abdul Rahman, which means it doesn't represent all young adults in Malaysia. This study collected the data through online questionnaire. Although there are explanations in it and before answering the questions, many participants are clueless when they are answering the questions. Inclusion and exclusion criteria have been attached with the questionnaire. However, some participants tend to answer the questionnaire before reading it thoroughly.

\section{CONCLUSION}

The prevalence of wrist pain is relatively high among young adults in Universiti Tunku Abdul Rahman, those who are having wrist pain according to (PRWE) score is $(71.53 \%, \mathrm{n}=98)$ and self-modified questionnaire is (22.92\%, $\mathrm{n}=33$ ). Prevention and awareness should be given to prevent wrist pain during the pandemic covid-19. Repetitive movement of the wrist and the ergonomics of the wrist are one of the few important risk factors which leads to wrist pain.

\section{Acknowledgement: None}

Conflict of Interest: None

Source of Funding: None

Ethical Approval: Approved 


\section{REFERENCES}

1. Barr AE. Work-Related Musculoskeletal Disorders of the Hand and Wrist: Epidemiology, Pathophysiology, and Sensorimotor Changes. Journal of Orthopaedic and Sports Physical Therapy. 2004;34(10):610-27.

2. Sharan D, Mohandoss M, Ranganathan R, Jose JA, Rajkumar JS. Distal upper extremity disorders due to extensive usage of hand held mobile devices. Annals of Occupational And Environmental Medicine. 2014;26(22):1041-5.

3. Lee. DL, McLoone. H, Dennerlein. JT. Observed finger behaviour during computer mouse use. Appl Ergon. 2008;39(1):107-13.

4. Rauf. W, Anwar. N, Ahmed A. Work Related Musculoskeletal Wrist Pain and Functional Disability in Office Workers Using Computer. Asian Journal of Allied Health Sciences (AJAHS). 2017;2(4):3640.

5. Ferguson R, Riley ND, Wijendra A, Thurley N, Carr AJ, Bjf D. Wrist pain: a systematic review of prevalence and risk factors- what is the role of occupation and activity? BMC Musculoskeletal Disorders. 2019;20(1).

6. Sansone C, Cohen J, Gold B, Zhu W, Misic AM, Beiting DP, et al. Aging-associated dysbiosis increases susceptibility to enteric viral infection in Drosophila. bioRxiv. 2017;
7. Loh PY, Yeoh WL, Nakashima H, Muraki S. Impact of keyboard typing on the morphological changes of the median nerve. Journal of Occupational Health. 2017; 59(5):408-17.

8. Stahl S, Vida D, Meisner C, Stahl AS, Schaller HE, Held M. Work related etiology of de Quervain's tenosynovitis: A casecontrol study with prospectively collected data Pathophysiology of musculoskeletal disorders. BMC Musculoskeletal Disorders. 2015;16(1):1-10.

9. Bain G, MacLean S, Yeo C, Perilli E, Lichtman D. The Etiology and Pathogenesis of Kienböck Disease. Journal of Wrist Surgery. 2016;05(04):e1-e1.

10. Alhusain FA, Almohrij M, Althukeir F, Alshater A, Alghamdi B, Masuadi E, et al. Prevalence of carpal tunnel syndrome symptoms among dentists working in Riyadh. Annals of Saudi Medicine. 2019; 39(2):104-11.

11. Phillips. N. Possible Causes of Wrist Pain and Treatment Tips. 2019.

How to cite this article: Selvakumar K, Sheng LY. The prevalence and risk factors of wrist pain among young adults in UTAR during movement control order: a cross-sectional study. Int J Health Sci Res. 2021; 11(11): 191-202. DOI: https://doi.org/10.52403/ijhsr.20211125 